Background: Lower urinary tract symptoms (LUTS) are an underdiagnosed but frequent manifestation in systemic sclerosis (SSc) [1]. LUTS pathogenesis in SSc is undetermined, mainly involving dysautonomia, fibrosis and a possible antibody-mediated damage [2]. Divergently from general population, female sex and advanced age are not reported to significantly impact LUTS in SSc [2].

Objectives: To evaluate the potential influence of gender and hormone-related factors in LUTS prevalence and severity among SSc patients (Pts).

Methods: A population of 42 SSc Pts and 50 age- and sex-matched healthy subjects (HSs) was evaluated. SSc diagnosis was based on 2013 ACR/EULAR criteria. Demographic data, medications interfering with pelvic floor dynamics and general comorbidities commonly associated with LUTS - diabetes mellitus, chronic heart failure, chronic obstructive pulmonary disease, peripheral neuropathy, pelvic organ prolapse, fecal incontinence - were recorded. Validated self-reported questionnaires derived from the International Conference on Incontinence were used to assess prevalence and severity of LUTS, namely of urinary incontinence (UI) and overactive bladder (OAB) [3]. Data were analysed using non-parametric tests. A $p$ value $<0.05$ and a confidence interval $(\mathrm{Cl})$ of $95 \%$ were considered statistically significant.

Results: There were no significant differences in main demographic data between SSc Pts and HSs. Specifically, median age was 61 years (IQR 21-85) vs 57 years (IQR 28-93) and female prevalence $83 \%$ vs $84 \%$ in SSc Pts vs HSs, respectively. Amongst the female population, $83 \%$ of SSc Pts vs $84 \%$ of HSs was in post-menopausal state, with a median of 1 (IQR 0-3) vs 1 (IQR 0-4) pregnancy by natural route, respectively. No woman of the study had received hormone replacement therapy or local hormonal therapies prior to the study. Similarly, there were not any significant differences in analysed comorbidities, while ongoing treatment was significantly different between the two populations, SSc patients more frequently receiving calcium channel blockers and glucocorticoids than healthy subjects $(p<0.001)$. In SSc Pts, statistically significant correlation was observed between stress UI and sex, with an increased female-to-male ratio $(p<0.005)$, but any significant difference was observed in US distribution depending on parity and menopausal state, nor on other analysed variables. Interestingly, female dominance has not resulted as a significant predictive factor for LUTS prevalence or severity in SSc Pts. In fact, in the regression analysis, SSc disease was the only significant predictor for LUTS (OR $3.45,95 \% \mathrm{Cl} 1.41-7.95 ; \mathrm{p}<0.01$ ), independently of other analysed variables, particularly of gender and hormone-related factors.

Conclusion: This study confirms the absence of pathogenic female-gender participation in LUTS prevalence among SSc Pts. However, consistently with findings on general population, a significant increased prevalence of urinary symptoms, particularly of stress UI, in SSc female Pts has emerged [4]. It is therefore conceivable that hormonal factors may act as a catalytic circumstance rather than pathogenic players in LUTS progression during SSc disease.

References:

[1] John G et al. Arthritis Care Res (Hoboken) 2018;70(8):1218-27

[2] John G. Clin Rheumatol. 2020;39(1):5-8

[3] Abrams P et al, J Urol. 2006;175:1063-6

[4] Abelson B et al. Biol Sex Differ. 2018;9(1):45

Disclosure of Interests: Greta Pacini: None declared, Sabrina Paolino: None declared, Federica Goegan: None declared, Pietro Francesco Bica: None declared, Elisa Alessandri: None declared, Carmen Pizzorni: None declared, Alberto Sulli Grant/research support from: Laboratori Baldacci, Emanuele Gotelli: None declared, Francesco Cattelan: None declared, Vanessa Smith Grant/research support from: The affiliated company received grants from Research Foundation - Flanders (FWO), Belgian Fund for Scientific Research in Rheumatic diseases (FWRO), Boehringer Ingelheim Pharma $\mathrm{GmbH} \& \mathrm{Co}$ and Janssen-Cilag NV, Consultant of: Boehringer-Ingelheim Pharma GmbH \& Co, Speakers bureau: Actelion Pharmaceuticals Ltd, Boehringer-Ingelheim Pharma $\mathrm{GmbH} \&$ Co and UCB Biopharma Sprl, Maurizio Cutolo Grant/research support from: Bristol-Myers Squibb, Actelion, Celgene, Consultant of: Bristol-Myers Squibb, Speakers bureau: Sigma-Alpha

DOI: 10.1136/annrheumdis-2020-eular.4701

\begin{tabular}{|l|l}
\hline THU0360 & EFFICACY OF A SELF-TREATMENT PROTOCOL \\
FOR FACE AND TEMPOROMANDIBULAR JOINTS \\
REHABILITATION IN SYSTEMIC SCLEROSIS (SSC)
\end{tabular}

M. Passalacqua ${ }^{1}$, C. Foggi ${ }^{2}$, N. Mauro ${ }^{2}$, L. Tofani ${ }^{3}$, S. Guiducci ${ }^{3}$, C. Bruni ${ }^{3}$, G. Lepri ${ }^{3}$, J. Blagojevic ${ }^{3}$, K. El Aoufy ${ }^{3}$, G. Fiori ${ }^{3}$, F. Bartoli $^{3}$, S. Maddali Bongi ${ }^{4}$, M. Mitola ${ }^{5}$, M. Gizduloch ${ }^{6}$, M. Matucci-Cerinic ${ }^{3}$, S. Bellando Randone ${ }^{3}$. ${ }^{1}$ Fondazione IRCCS Don Carlo Gnocchi ONLUS, Florence, Italy; ${ }^{2}$ University of Florence, Florence, Italy; ${ }^{3}$ Department of Experimental and Clinical Rheumatology, University of Florence, Dept. Geriatric Medicine, Div. of Rheumatology, Florence, Italy; ${ }^{4}$ Associazione Multidisciplinare Riabilitazione
Reumatologica AMURR, Florence, Italy; ${ }^{5}$ ASSMAF ONLUS, Florence, Italy; ${ }^{6}$ Associazione Italiana Gnatologia, Florence, Italy

Background: In SSc, skin involvement of the face is frequent and extremely disabling, resulting in limited mouth opening, an altered dentition, difficulty in teeth care, as well as having a strong impact on the emotional and psychological well-being, thus impairing quality of life.

Objectives: to evaluate the efficacy of a self-treatment protocol (created by AMURR A Multidisciplinary Association of Rheumatological Rehabilitation) for face and tempomandibular joints (TMJs) rehabilitation with two devices used in the dental field.

Methods: 40 SSc patients (37 female and 3 male) with a mouth opening $\leq 40 \mathrm{~mm}$, were recruited and randomized in two groups of treatment: Group 1 (20 patients: mean age 50,650 yrs $\pm 13,937 \mathrm{SD}$, mean disease duraton 10,45 yrs $\pm 7,877 \mathrm{SD}$, opening mouth $32,250 \mathrm{~mm} \pm 5,590 \mathrm{SD}$ ) treated with a home self-treatment protocol consisting of 23 exercises carried out at home in front of a mirror, 22/23 exercises were performed once a day, one of these using a device to obtain uniform stretching of the buccal rhyme, another one usingused three times a day to reduce tension of muscles of the TMJs, facilitating the mouth opening; group 2 (20 patients: mean age 58,05 yrs $\pm 18,103$ SD, mean disease duration 17,4 yrs $\pm 15,017 \mathrm{SD}$, opening mouth $34,950 \mathrm{~mm} \pm 5,753$ ) without physical rehabilitation, only drugs as treatments of SSc and its complications. All patients underwent a baseline (T0) and 45 days (T1) clinimetric assessment by self-assessment of quality of life with SF-36 (Short-Form 36 Health Survey), of the degree of disability of the mouth with MHISS (of the Mouth Handicap in Systemic Sclerosis scale), Muscle pain evaluated by numerical rating scale (NRS) of the temporomandibular joint with TMD (Temporo mandibular Disorders), evaluation of mouth opening and ROM of the cervical spine. Statistical analysis was performed using the t-test or the Mann-Whitney test for assessing changes in all measurement scales between treatment groups.

Results: The protocol of home physiotherapy exercises resulted in a statistically significant improvement in the treated group compared to group 2 both for mouth opening (T0: $32,250 \pm 5,590, \mathrm{~T} 1: 35,650 \pm 6,046)$ vs (T0: $34,950 \pm 5,753 \mathrm{~T} 1$ : $34,300 \pm 6,001)(p<0.001)$, cervical flexion (T0: $2,950 \pm 1,939$ T1: $1.700 \pm 1,525)$ vs (T0: $4,450 \pm 2,282 \mathrm{~T} 1: 4,075 \pm 2,238)(\mathrm{p}<0.01)$, cervical extension (T0: 17,025 $\pm 1,895 \mathrm{~T} 1: 17,625 \pm 1,605)$ vs $(\mathrm{T} 0: 17,050 \pm 2,089 \mathrm{~T} 1: 16,525 \pm 3,110)(p<0.05)$ cervical right lateral flexion (T0: 14,075 $\pm 2,386$ T1:13,400 $\pm 2,431$ ) vs (T0: 14,200 $\pm 1,765 \mathrm{~T} 1: 14,425 \pm 1,742)(p<0.01)$, cervical right rotation (T0: 14,200 $\pm 3,416$ $\mathrm{T} 1: 13,750 \pm 3,206)$ vs (T0: 14,900 $\pm 1,683 \mathrm{~T} 1: 15,550 \pm 2,188)(\mathrm{p}<0.01)$, cervical left rotation (T0: 14,725 $\pm 3,640$ T1:14,450 \pm 3,710) vs (T0: $15,900 \pm 2,614$ T1 $16,450 \pm 2,964)(p<0.05)$, mouth disability at MHISS (T0: 19,100 $\pm 10,356$ T1: $16,000 \pm 9,989)$ vs (T0: $20,950 \pm 9,950, T 1: 21,100 \pm 10,775)(p<0.01)$.

Conclusion: The use of the home exercises protocol associated with the two devices has shown a significant improvement of the disability linked to skin involvement of the face. This highlights the fundamental role that home rehabilitation self therapy has in practice. These data will need to be confirmed in a larger cohort of patients

Disclosure of Interests: Mauro Passalacqua: None declared, Cristian Foggi: None declared, Nicola Mauro: None declared, Lorenzo Tofani: None declared, Serena Guiducci: None declared, Cosimo Bruni Speakers bureau: Actelion, Eli Lilly, Gemma Lepri: None declared, Jelena Blagojevic: None declared Khadija El Aoufy: None declared, Ginevra Fiori: None declared, Francesca Bartoli: None declared, Susanna Maddali Bongi: None declared, Marco Mitola: None declared, Marco Gizduloch: None declared, Marco Matucci-Cerinic Grant/research support from: Actelion, MSD, Bristol-Myers Squibb, Speakers bureau: Acetelion, Lilly, Boehringer Ingelheim, Silvia Bellando Randone: None declared

DOI: 10.1136/annrheumdis-2020-eular.3451

\section{THU0361 \\ EPIDEMIOLOGIC VARIATION ON SCLERODERMA RENAL CRISIS AND CLINICAL FEATURES VARIATION ON SYSTEMIC SCLEROSIS PATIENTS OVER TIME: DATA FROM RESCLE REGISTRY.}

X. Pla Salas ${ }^{1}$, C. Tolosa ${ }^{2}$, A. Guillén del Castillo ${ }^{3}$, M. E. Sánchez García ${ }^{4}$, J. Sánchez-Redondo ${ }^{5}$, E. L. Callejas-Moraga ${ }^{2}$, L. Sáez-Comet ${ }^{6}$, J. A. VargasHitos $^{7}$, J. A. Todolí Parra ${ }^{8}$, L. Trapiella Martínez ${ }^{9}$, I. Rodriguez-Pubto ${ }^{10}$, M. Freire ${ }^{11}$, I. Pons Martin del Campo ${ }^{1}$, V. Fonollosa-Pla ${ }^{3}$, C. P. Simeón-Aznar ${ }^{3}$, O. B. O. R. I. Autoimmune Diseases Study Group (Geas) ${ }^{12}$. ${ }^{1}$ Xarxa Assistencial Universitaria Althaia, Internal Medicine, Manresa, Spain; ${ }^{2}$ Corporación Sanitaria Universitaria Parc Taulí, Internal Medicine, Sabadell, Spain; ${ }^{3} \mathrm{Hospital}$ Universitario Vall d'Hebron, Unit of Autoimmune Diseases. Internal Medicine., Barcelona, Spain; ${ }^{4}$ Hospital Universitario Virgen de Valme, Internal Medicine, Sevilla, Spain; ${ }^{5}$ Hospital Universitario de Móstoles, Internal Medicine, Móstoles, Spain; ${ }^{6}$ Hospital Universitario Miguel Servet, Internal Medicine, Zaragoza, Spain; ${ }^{7}$ Hospital Universitario Virgen de las Nieves, Internal Medicine, Granada, 
Spain; ${ }^{8}$ Hospital Universitario y Politécnico La Fe, Internal Medicine, Valencia, Spain; ${ }^{9}$ Hospital de Cabueñes, Unit of Systemic Autoimmune Diseases. Internal Medicine., Gijón, Spain; ${ }^{10}$ Hospital Universitario Mútua Terrassa, Unit of Autoimmune Diseases. Internal Medicine., Terrassa, Spain; ${ }^{11}$ Hospital Clínico Universitario de Santiago, Unit of Autoimmune Diseases. Internal Medicine., Santiago de Compostela, Spain; ${ }^{12}$ Sociedad Española de Medicina Interna (SEMI), Madrid, Spain

Background: Scleroderma renal crisis (SRC) prevalence is decreasing. However, no Systemic Sclerosis (SSc) patient's registry has evaluated that decrease over time. No treatment have been able to prevent SRC development.

Objectives: Primary objective: to identify SRC prevalence in 2 periods in the RESCLE (Registro de ESCLErodermia) registry. Secondary objective: to idenfy which features could justify that change on SRC prevalence.

Methods: Up to December 2018, 1937 SSc patients were included by 31 referral centers in RESCLE registry. SRC prevalence and incidence in diagnosed patients before and after 2003 was determined. Clinical characteristics of diagnosed patients in each period of time were analysed to identify differences between them.

Results: Out of 1937 SSc, 43 (2.2\%) developed SRC. Prevalence of SRC before and after 2003 was $3.5 \%$ and $1.08 \%$. SRC Incidence: Graphic 1. Significant differences between Pre-2003 vs. Post-2003 SSc cohorts were found in univariate analysis: Table 1 and 2.

Table 1. Univariate analysis

\begin{tabular}{lll}
\hline & Pre-2003(\%) & Post-2003(\%) \\
\hline Demographic data & 63 & \\
IcSSc & 28 & 59 \\
dcSSc & 6.4 & 15 \\
SSSSc & 1.1 & 3.3 \\
Early SSc & 1.9 & 9.6 \\
Very early SSc & $49.1( \pm 15.2) y$ & $55.0( \pm 15.6) y$ \\
Age at SSc dx & $1.1(0.2-4.3) y$ & $0.6(0.1-1.5) y$ \\
Time from SSc dx to SRC & 99 & 86 \\
ACR/EULAR 2013 criteria & 20 & 37 \\
Comorbidities & 35 & 29 \\
Smoked & & 25 \\
Arterial hypertension & 38 & 19 \\
Diagnostic procedures & 8.9 & 4.8 \\
PAPs $>$ 40mmHg by Echocardiography & 12 & 11 \\
Not-sclerodermal pattern at VCS & & 1.6 \\
Lupus anticoagulant & 29 & \\
Prognostic features & 10 & \\
Overall mortality & & \\
ILD-related death & & \\
\hline
\end{tabular}

Conclusion: SRC Prevalence and Incidence has decreased. Prevalence is three-fold in diagnosed SSc cohort pre-2003 than in post-2003. The post-2003 cohort showed lesser prevalence of dcSSc subtype, earlier SSc diagnosis, less organic involvement and more intensive treatment than pre-2003 cohort. All these findings could explain the decline in the SRC prevalence.

Figure:

\section{SRC incidence: before and after 2003}

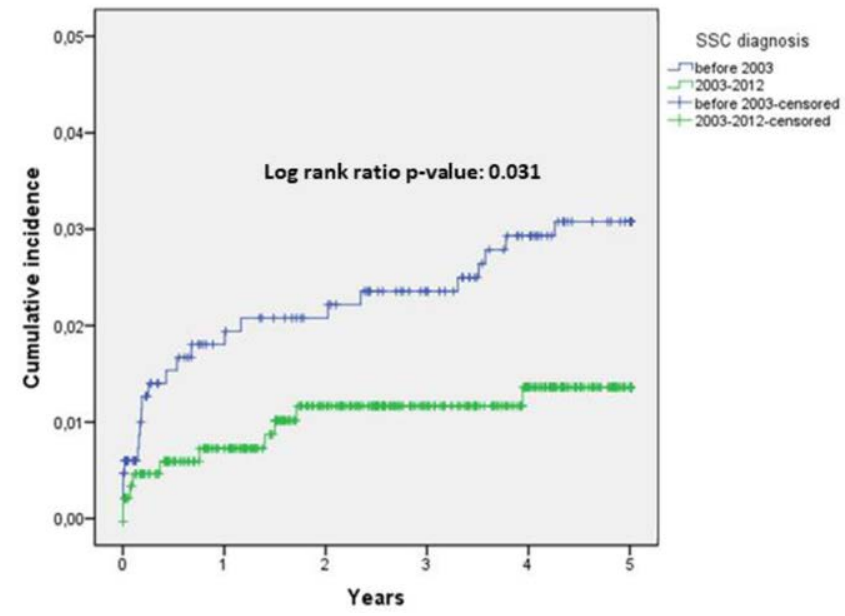

Table 2. Univariate analysis

\begin{tabular}{lcc}
\hline & Pre-2003 (\%) & Post-2003 (\%) \\
\hline Clinical data & 7.2 & \\
Skin sclerosis (as $1^{\text {st }}$ symptom) & 49 & 4.1 \\
Digital ulcers & 66 & 31 \\
Telangiectasia & 12 & 52 \\
Acroosteolysis & 29 & 4.6 \\
Calcinosis & 28 & 14 \\
Joint contractures & 49 & 13 \\
ILD & 20 & 37 \\
Cardiac conduction alteration & 41 & 27 \\
Left diastolic dysfunction & 13 & 32 \\
Peripheral neuropathy & 37 & 6.1 \\
Sicca syndrome & & 24 \\
Treatment features & 23 & 40 \\
Calcium channel blockers & 2.1 & 14 \\
Specific vasodilators & 0.99 & 3.4 \\
Prostaglandin & 5.1 & 16 \\
Angiotensin system inhibitors & 11 & 20 \\
Corticosteroids & 3 & 15 \\
Immunosuppressant & &
\end{tabular}

y: years old; IcSSc: limited cutaneous SSc; dcSSc: diffuse cutaneous SSc; ssSSc: sine scleroderma SSc; ILD: Interstitial lung disease; PAPs: systolic Pulmonary Arterial Pressure; VCS: Video-capillaroscopy; dx: diagnosis

Disclosure of Interests: Xavier Pla Salas: None declared, Carles Tolosa Consultant of: Actelion pharmaceuticals, GSK, MSD., Alfredo Guillén del Castillo: None declared, María Esther Sánchez García: None declared, Jorge Sánchez-Redondo: None declared, Eduardo L. Callejas-Moraga: None declared, Luis Sáez-Comet: None declared, Jose Antonio Vargas-Hitos: None declared, Jose Antonio Todolí Parra: None declared, Luis Trapiella Martínez: None declared, Ignasi Rodriguez-Pubto: None declared, Mayka Freire: None declared, Isaac Pons Martin del Campo: None declared, Vicent Fonollosa-Pla Consultant of: Actelion pharmaceuticals, GSK, MSD., Carmen Pilar Simeón-Aznar Consultant of: Actelion pharmaceuticals, GSK, MSD., on behalf of RESCLE Investigators, Autoimmune Diseases Study Group (GEAS): None declared

DOI: 10.1136/annrheumdis-2020-eular.1353

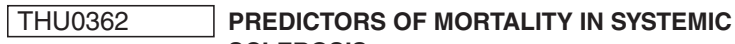 SCLEROSIS}

S. DE Almeida Chaves ${ }^{1}$, T. Porel ${ }^{1}$, D. Adoue ${ }^{1}$, L. Astudillo ${ }^{1}$, D. Ribes ${ }^{2}$, G. Prévot ${ }^{3}$, F. Gaches ${ }^{4}$, L. Alric ${ }^{1}$, L. Sailler ${ }^{1}$, G. Pugnet ${ }^{1} .{ }^{1}$ Centre Hospitalier Universitaire, Medecine Interne, Toulouse, France; ${ }^{2}$ Centre Hospitalier Universitaire, Néphrologie, Toulouse, France; ${ }^{3}$ Centre Hospitalier Universitaire, Pneumologie, Toulouse, France; ${ }^{4}$ Hôpital Joseph Ducuing, Medecine Interne, Toulouse, France

Background: Systemic sclerosis (SSc) is known as the most severe connective tissue disorder, and to be associated with a high mortality risk. Moreover, there is a great variability of mortality rate in the literature.

Objectives: To determine the mortality rate and its predictors in systemic sclerosis in a long-term follow- up of a bi-centric cohort of SSc patients

Methods: We have carried out a retrospective observational study by systematically analysing the medical records of patients diagnosed with SSc in Toulouse University Hospital and Ducuing Hospital. We included patients over 18 years old, whose diagnosis was made after 01/01/2000 and who met the classification criteria for the American College of Rheumatology (ACR) /EULAR 2013, and with at least one follow-up visit after the inclusion visit. Patients were followed until 30/05/2018. We quantified mortality as Standardized Mortality Ratio (SMR) and percentage mortality at $1,3,5,10$ and 15 years of disease. For comparison, we used the mortality rate for the population in the French Region of Occitanie in 2016, using data from the French National Statistical Agency. We determined a single primary cause of death (SSc or non-SSc related) and evaluated predictors of mortality using multivariable Cox regression.

Results: We included 375 patients (292 females), 63 (15.2\%) patients with diffuse cutaneous SSc, 279 (76.7\%) with limited cutaneous SSc and $33(8.1 \%)$ with sine scleroderma SSc. During the study period 6 patients were lost to follow-up and 69 died (18.4\%). The SMR ratio was 1.85 (95\% Cl 1.45-2.34). The overall survival rates were $97.5 \%$ at 1 year, $93.4 \%$ at 3 years, $87.1 \%$ at 5 years, $77.9 \%$ at 10 years and $61.3 \%$ at 15 years. Among the 69 deaths recorded $46.4 \%$ were SSc related deaths, $53.6 \%$ were non-related. Among the SSc related deaths, three main causes were identified: interstitial lung disease (ILD) $(34.4 \%)$, pulmonary hypertension $(31.2 \%)$ and digestive tract involvement $(18.8 \%)$. Among the causes of SSc non-related deaths, cardiovascular 